Document downloaded from:

http://hdl.handle.net/10251/83222

This paper must be cited as:

Hernández Mejías, MA.; Sala Piqueras, A.; Arino, C.; Querol, A. (2016). Reliable controllable sets for constrained Markov-Jump Linear Systems. International Journal of Robust and Nonlinear Control. 26(10):2075-2089. doi:10.1002/rnc.3394.

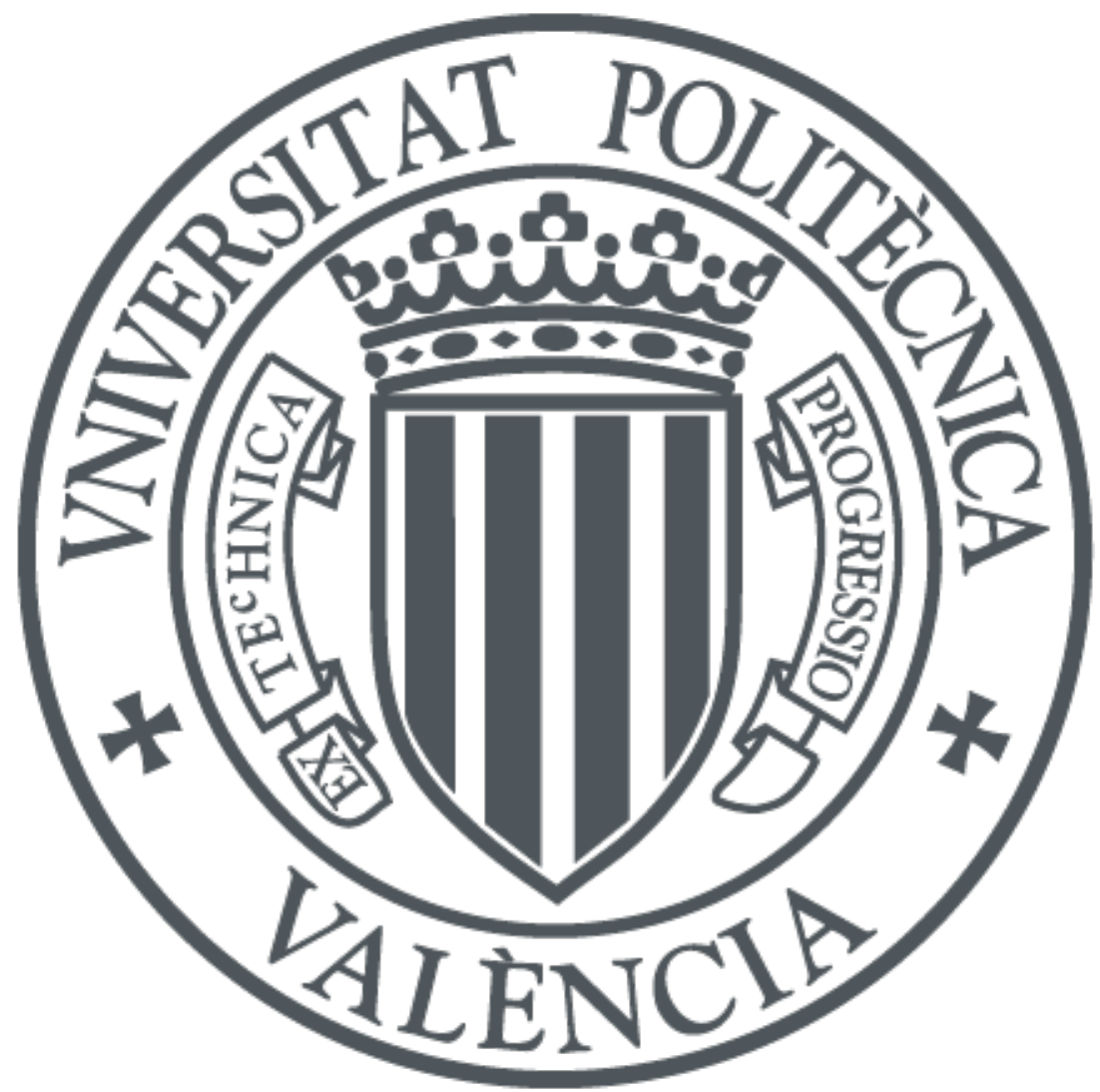

The final publication is available at

http://doi.org/10.1002/rnc.3394

Copyright Wiley

Additional Information 


\title{
Reliable controllable sets for constrained Markov-Jump Linear Systems
}

\author{
Manuel A. Hernández-Mejías ${ }^{1, * \dagger}$, Antonio Sala ${ }^{1}$, Carlos Ariño $^{2}$ and Andrés Querol ${ }^{2}$ \\ ${ }^{1}$ Dept. Ing. Sistemas y Automática. Universitat Politècnica de València. Cno Vera s/n, E-46022 Valencia, Spain. \\ ${ }^{2}$ Dept. Sistemas Industriales y Diseño. Universitat Jaume I. Av. Vicent Sos Baynat, s/n E-12071 Castellón, Spain.
}

\begin{abstract}
SUMMARY
Robust $\lambda$-contractive sets have been proposed in previous literature for uncertain polytopic linear systems. It is well known that, if initial state is inside such sets, it is guaranteed to converge to the origin. This work presents the generalization of such concepts to systems whose behaviour changes among different linear models with probability given by a Markov chain. We propose sequence-dependent sets and associated controllers which can ensure a reliability bound when initial conditions are outside the maximal $\lambda$ contractive set. Such reliability bound will be understood as the probability of actually reaching the origin from a given initial condition without violating constraints. As initial condition are further away from the origin, the likelihood of reaching the origin decreases. Copyright (c) 2015 John Wiley \& Sons, Ltd.
\end{abstract}

Received ...

KEY WORDS: Constrained linear systems; Invariant sets; Fault-tolerant control; Markov-Jump Linear Systems; Reliability analysis. Int. J. Robust. Nonlinear Control

\section{INTRODUCTION}

Control of constrained systems needs assessing the set of initial conditions which can be steered to the origin without violating such constraints during the transient. To that purpose, robust $\lambda$ contractive controllable sets have been proposed in priori literature for uncertain polytopic linear systems [1, 2, 3, 4], as well as for non-linear ones, based, for instance, in the linear polytopic embedding [5]. It is well known that, if initial state is inside such sets, it is guaranteed to converge to the origin with a particular geometric contraction rate $\lambda$. Switching systems can also be considered into this framework; for instance, [6] extends the above ideas to dwell-time constrained switching. If state and input constraint sets are polyhedral, the sets in the proposed algorithms can be computed with well known algorithms, such as those in the multi-parametric toolbox (MPT) [7, 8].

When probability information is incorporated, in some cases, uncertainty can be modelled as a set of discrete-time linear systems (denoted as “modes") with mode transitions governed by a Markov

\footnotetext{
*Correspondence to: Manuel A. Hernández-Mejías, Dept. Ing. Sistemas y Automática. Universitat Politècnica de València. Cno Vera s/n, E-46022 Valencia, Spain.

†Email: maher3@upv.es

Copyright (C) 2015 John Wiley \& Sons, Ltd.

Prepared using rncauth.cls [Version: 2010/03/27 v2.00]
} 
chain; such systems, exhibiting random abrupt changes in their structure, are usually denoted as Markov-jump linear systems [9]. Practical applications of such modelling framework arise in, for instance, networked control systems [10, 11], manufacturing systems [9] and, particularly, faulttolerant control set-ups $[12,13,14,15]$.

There is a considerable amount of published studies describing unconstrained control strategies for MJLSs [9, 16, 17]. Control of constrained MJLS has been addressed in an LMI framework $[18,19,20,21]$. Obviously, the proven domain of attraction of such controllers must lie into the maximal robust $\lambda$-contractive sets above discussed.

Reliability analysis of a control strategy in presence of mode changes (faults) is a problem of significant interest, motivating this work. Indeed, problems arise when initial conditions are outside the proven maximal sets. In such a case, the controller might stabilize the system without constraint violation only for some mode sequences, i.e., with probability lower than 1 .

This work focuses on the above issue, proposing an algorithm which determines sequencedependent sets for which there exists a control law such that the system can be steered to the origin, but only for a particular sequence. If the algorithm converges, then, states outside such sets cannot be driven to the origin without violating constraints, whichever the mode sequence: the likelihood of reaching the origin from them is zero. Obviously, initial conditions in a robust $\lambda$-contractive controllable set have $100 \%$ likelihood of reaching the origin. If the state lies in "intermediate" regions, this work proposes a control law which will steer the state to the origin with a certain probability bound $\underline{p}$, to be denoted as reliability bound.

Two settings will be considered regarding the mode information available to the controller at the moment of computing the control action in on-line operation. The first one will discuss the case in which the current mode is available; the second one will discuss controllers without such mode information. The former is a widely used assumption in control of MJLS (present in most of the above-cited works); the latter (no mode information) will yield controllers with lower reliability, as intuitively expected.

The structure of the paper is as follows: next section will discuss preliminary definitions and notation; Section 3 details the generalization of well-known one-step and $l$-step sets to sequencedependent ones. Then, Section 4 proposes a state-feedback controller based on the computed sets with a certain minimum reliability bound. Section 4.3 discusses implementation and computational load. A numerical example in Section 5 illustrates the results. A conclusion section closes the paper.

\section{PRELIMINARIES, NOTATION AND PROBLEM STATEMENT}

Consider a non-autonomous linear time-varying system, for $k \geq 0$ :

$$
x_{k+1}=A_{\theta_{k}} x_{k}+B_{\theta_{k}} u_{k}, \quad \theta_{k} \in \mathcal{M},
$$

where $x_{k} \in \mathbb{R}^{n}$ represents the state vector, $u_{k} \in \mathbb{R}^{m}$ the control actions, being $\mathcal{M}=\{1,2, \ldots M\}$ a set of possible modes, so the system (1) switches between $M$ different operation modes, i.e.,

$$
A_{\theta_{k}} \in \overline{\mathcal{A}}=\left\{A_{1}, \ldots, A_{M}\right\}, \quad B_{\theta_{k}} \in \overline{\mathcal{B}}=\left\{B_{1}, \ldots, B_{M}\right\}
$$


In this paper, it will be assumed that the mode parameter $\theta_{k}$ follows a discrete-time Markov chain with transition probabilities matrix $\mathbb{P}=\left[\pi_{j i}\right]$, i.e., $\operatorname{Pr}\left\{\theta_{k+1}=j \mid \theta_{k}=i\right\}=\pi_{i j}$, were $\pi_{j i} \geq 0$,

$\forall i, j \in M$ and $\sum_{j=1}^{M} \pi_{j i}=1$. Apart from parameter jumps, the mode matrices will be assumed time-invariant ( $\overline{\mathcal{A}}$ and $\overline{\mathcal{B}}$ are constant) and $\pi_{j i}$ will not change with time.

The current mode $\theta_{k}$ can be either known at the instant of computing $u_{k}$ or not. That will give rise to different control action possibilities later in this work.

\subsection{Robust l-step sets}

In the case there is no mode information available to the controller, system (1) can be considered to be an uncertain linear system with polytopic uncertainty. In that context, let us assume the state must be constrained into a region $\Omega$ and that the control action must, too, belong to a known set $\mathbb{U}$.

The following definition is well known:

Definition 1 (Robust 1-step set [2,4])

$$
Q(\mathcal{C}):=\left\{x \in \mathbb{R}^{n}: \exists u \in \mathbb{U} \text { such that } A_{i} x+B_{i} u \in \mathcal{C} \forall i \in \mathcal{M}\right\}
$$

With the above definition, following [4], given a contraction rate $0 \leq \lambda \leq 1$, the recursivelydefined set:

$$
\mathcal{K}_{l}^{\lambda}=Q\left(\lambda \mathcal{K}_{l-1}^{\lambda}\right) \cap \Omega
$$

starting with $\mathcal{K}_{0}^{\lambda}=\Omega$, will be denoted as the robust $l$-step $\lambda$-contractive controllable set. Indeed, as shown in the cited reference, for all $x_{0} \in \mathcal{K}_{l}^{\lambda}$ there exists an admissible control action $\boldsymbol{u}$ which, without knowledge of the actual mode, keeps the future states inside $\Omega$ at least $l$ time steps, and $x_{k}^{\boldsymbol{\theta}}\left(x_{0}, \boldsymbol{u}\right) \in \lambda^{k} \Omega$ for all $\theta \in \mathcal{M}^{l}$.

When super-index $\lambda$ is omitted, it will be assumed to take the value of $\lambda=1$, denoting the socalled invariant sets. Such convention $(\lambda=1)$ when omitted will also be used in later variations of the above invariant concepts.

If the iteration (4) converges for $l \rightarrow \infty$, the resulting set will be labelled as $\mathcal{K}_{\infty}^{\lambda}$. This is largest set of initial states in $\Omega$ for which there exists a mode-independent controller making $\mathcal{K}_{\infty}^{\lambda}$ to be $\lambda$-contractive (maximal $\lambda$-contractive controllable set), see [4] for details.

Obviously, if the initial state belongs to the above maximal robust $\lambda$-contractive sets, it will robustly converge to the origin (irrespective of the mode sequence, without information on current mode).

\subsection{Problem statement and notation}

The objective of this work is extending the above $\lambda$-contractiveness concepts to initial states outside the maximal robust set. Obviously, such states will not converge to the origin under some sequences; this work will propose suitable control laws for them, and will estimate the probability of success in driving the state to the origin.

Two information scenarios (either known or unknown current mode) will be considered. When current mode is known to the controller, a more flexible definition of $\mathcal{Q}(\mathcal{C})$ will be proposed, allowing for mode-dependent $u^{i}$ in (3), and a higher probability of success will be guaranteed. 
In order to address the problem with more generality, we will consider mode-dependent constraints, as well as the possibility of some transitions having zero probability to enlarge the obtained guaranteed sets.

The mode-dependent constraints and essential notation to be used throughout the paper will be defined next.

Constraints. Some mode-dependent state and input constraints will be considered. In particular, a list of sets $\bar{\Omega}:=\left\{\Omega^{[1]}, \ldots, \Omega^{[M]}\right\}, \bar{U}:=\left\{\mathbb{U}^{[1]}, \ldots, \mathbb{U}^{[M]}\right\}$ will be specified as the constraint for each mode ( $x_{k}$ must belong to $\Omega^{\left[\theta_{k}\right]}$, and $u_{k}$ must belong to $\mathbb{U}^{\left[\theta_{k}\right]}$ ). The origin $x=0$ will be assumed to belong to all $\Omega^{[i]}$, and likewise $u=0$ will be assumed to belong to all $\mathbb{U}^{[i]}$, for all $i \in \mathcal{M}$.

The notation $\Omega^{*}$ will denote the set in the augmented space $\mathbb{R}^{n} \times \mathcal{M}$ given by $\Omega^{*}:=\{(x, \theta) \in$ $\left.\mathbb{R}^{n} \times \mathcal{M}: x \in \Omega^{[\theta]}\right\}$. Hence, the mode-dependent constraint can be stated, for all $k \geq 0$, as $\left(x_{k}, \theta_{k}\right) \in \Omega^{*}$, and $\left(u_{k}, \theta_{k}\right) \in \mathbb{U}^{*}$ being $\mathbb{U}^{*}$ likewise defined. Analogously, for any set $\mathcal{C}^{*} \subset \Omega^{*}$, the "cuts" for a particular mode will be understood as $\mathcal{C}^{[\theta]}:=\left\{x \in \Omega^{[\theta]}:(x, \theta) \in \mathcal{C}^{*}\right\}$. Given an arbitrary set $\mathcal{C} \in \mathbb{R}^{n}$, the notation $\lambda \mathcal{C}$ will refer to a linear scaling of $\mathcal{C}$ with scaling factor $0 \leq \lambda \leq 1$. Given $\mathcal{C}^{*} \subset \Omega^{*}$, the scaling $\lambda \mathcal{C}^{*}$ will be understood as $\lambda \mathcal{C}^{*}:=\left\{(x, \theta): x \in \lambda \mathcal{C}^{[\theta]}\right\}$.

We will denote as $\Omega=\cap_{i} \Omega^{[i]}, \mathbb{U}=\cap_{i} \mathbb{U}^{[i]}$ which will be "safe" sets fulfilling the constraints for all modes. If no mode information is available when computing the control action, the controller should obviously assume single $\Omega$ and $\mathbb{U}$ as state and input constraints (mode-independent), as considered in Section 2.1.

Sequence notation. Using repeated Cartesian product, we will denote as $\mathcal{M}^{l}$, the set of all possible mode sequences of length $l>0$. Expression $\operatorname{len}(\boldsymbol{\theta})$ will denote the length of sequence $\boldsymbol{\theta}$, i.e., if $\boldsymbol{\theta} \in \mathcal{M}^{l}$, then $\operatorname{len}(\boldsymbol{\theta})=l$. Bold symbols $\boldsymbol{\theta}$ will be used to denote generic sequences, whereas non-bold ones, say $\psi$, will denote single sequence elements -equivalently, length-1 sequences-.

For a particular $\boldsymbol{\theta} \in \mathcal{M}^{l}, \boldsymbol{\theta}=\left\{\theta_{0}, \theta_{1}, \ldots, \theta_{l-1}\right\}$, denote by $\boldsymbol{\theta}_{i: j}$, for $0 \leq i \leq j \leq l-1$, the subsequence $\left\{\theta_{i}, \theta_{i+1}, \ldots, \theta_{j}\right\} \in \mathcal{M}^{j-i+1}$. Given $\boldsymbol{\eta} \in \mathcal{M}^{l_{1}}, \boldsymbol{\xi} \in \mathcal{M}^{l_{2}}$, denote as $\boldsymbol{\eta} \boldsymbol{\xi}$ the concatenated sequence $\left\{\eta_{0}, \ldots, \eta_{l_{1}-1}, \xi_{0}, \ldots, \xi_{l_{2}-1}\right\} \in \mathcal{M}^{l_{1}+l_{2}}$.

Given the above $\boldsymbol{\theta}, \operatorname{Pr}(\boldsymbol{\theta})$ will denote the probability of $\boldsymbol{\theta}$, i.e., $\operatorname{Pr}(\boldsymbol{\theta})=\operatorname{Pr}\left(\theta_{0}\right) \Pi_{h=1}^{l-1} \pi_{\theta_{h} \theta_{h-1}}$ where $\operatorname{Pr}\left(\theta_{0}\right)$ is obtained with the available on-line information at a particular moment.

A sequence $\boldsymbol{\theta}$ will be said to be admissible if $\Pi_{h=1}^{l-1} \pi_{\theta_{h} \theta_{h-1}} \neq 0$. Given the transition probabilities, the so-called incidence matrix $\mathcal{I}$ will be defined as the one whose element at position $(i, j)$ is equal to 1 if $\pi_{i j} \neq 0$, and equal to zero otherwise.

The set of admissible sequences of length $l$ will be denoted as $\overline{\mathcal{M}}^{l}$. Evidently, a sequence is admissible if and only if all its sub-sequence are, too, admissible. Given a particular mode, its admissible successors will be denoted by $S(i):=\left\{j: \pi_{j i} \neq 0\right\}$. Of course, if $S(i)=\mathcal{M}$ for all $i$, then any arbitrary switching is admissible.

Note that if sequence switching has a minimum dwell-time constraint, such as the case considered in [6], it can be trivially considered the output of a Markov process with a larger number of states, so the results to be presented here can also be applied to such a case. 
Sets associated to sequences. The notation $\boldsymbol{u} \in \mathbb{U}_{\boldsymbol{\theta}}, \boldsymbol{u}=\left\{u_{0}, u_{1}, \ldots, u_{l-1}\right\}$ will be used for input sequences of length $l$ such that constraints are not violated, i.e., $u_{k} \in \mathbb{U}^{\left[\theta_{k}\right]}$. An analogous notation to state sequences $\boldsymbol{x} \in \Omega_{\boldsymbol{\theta}}$ will be used, too.

Predicted state sequences. Given a mode sequence $\boldsymbol{\theta}$, for the controlled system (1), with an input sequence $\boldsymbol{u} \in \mathbb{U}_{\theta}$, the associated state sequence $\boldsymbol{x}^{\boldsymbol{\theta}}$ will be defined as:

$$
x_{0}, x_{1}^{\boldsymbol{\theta}}:=A_{\theta_{0}} x_{0}+B_{\theta_{0}} u_{0}, x_{2}^{\boldsymbol{\theta}}:=A_{\theta_{1}} x_{1}^{\boldsymbol{\theta}}+B_{\theta_{1}} u_{1}, \ldots, x_{l}^{\boldsymbol{\theta}}:=A_{\theta_{l-1}} x_{\theta_{l-1}}^{\boldsymbol{\theta}}+B_{\theta_{l-1}} u_{l-1},
$$

where, actually $x_{k}^{\boldsymbol{\theta}}$ is a function $x_{k}^{\boldsymbol{\theta}}\left(x_{0}, \boldsymbol{u}\right)$, in fact, by causality, only of $x_{k}^{\boldsymbol{\theta}}\left(x_{0}, \boldsymbol{u}_{0: k-1}\right)$.

An input sequence (and the associated predicted state trajectory) will be denoted as admissible for initial state $x_{0}$ and sequence $\boldsymbol{\theta}$ if mode-dependent constraints are not violated, i.e., if $\boldsymbol{u} \in \mathbb{U}_{\boldsymbol{\theta}}$ and $\boldsymbol{x}^{\boldsymbol{\theta}}\left(x_{0}, \boldsymbol{u}\right) \in \Omega_{\boldsymbol{\theta}}$.

\section{AUGMENTED AND SEQUENCE-DEPENDENT SETS}

In this section, generalizations of (3) will be proposed. First, the extension to the case when the controller knows the current operating mode and the admissible successors. Later, sequencedependent sets will be defined so that, in Section 4, the knowledge of the probabilities of future sequences will allow the controller to "bet" over the most likely outcome to decide the course of action.

\subsection{Augmented l-step sets}

Let us redefine the one-step set in the augmented space $\Omega^{*}$ as:

$$
Q^{*}\left(\mathcal{C}^{*}\right):=\left\{\left(x_{0}, i\right) \in \Omega^{*}: \exists u \in \mathbb{U}^{[i]} \text { such that }\left(A_{i} x_{0}+B_{i} u, j\right) \in \mathcal{C}^{*} \forall j \in S(i)\right\},
$$

where knowledge of the mode $i$ by the controller is implicitly embedded in the definition. Then, the iterative algorithm for $l$-step sets can be rewritten as Algorithm 1 .

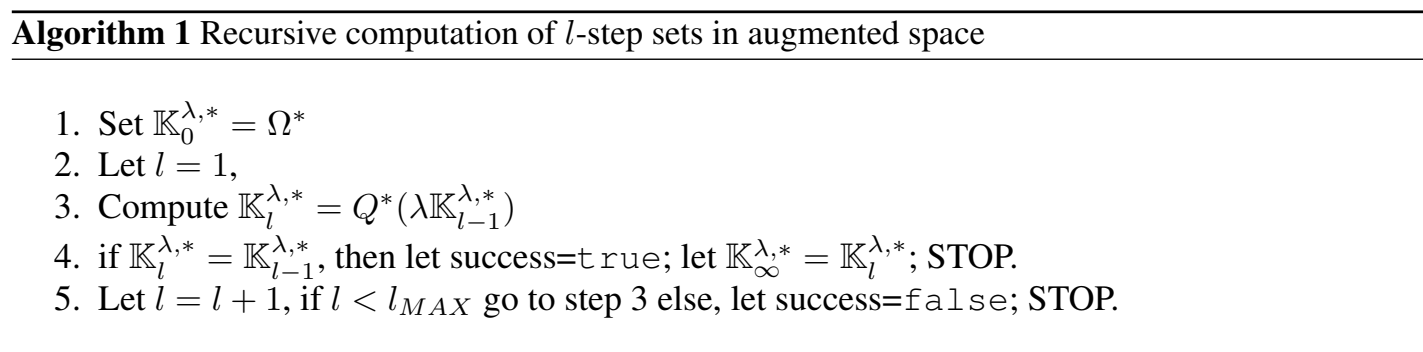

\section{Theorem 1}

If Algorithm 1 converges (success=true), the set $\mathbb{K}_{\infty}^{\lambda, *}$ is the maximal $\lambda$-contractive controllable set contained in $\Omega^{*}$. 
Proof

Omitted, as would be almost identical to those in the non-augmented space [2, Theorem 3.1],[4].

\section{Remark 1}

Convergence of the algorithm in a finite number of steps cannot be guaranteed in general. However, if there exists any $\lambda$ such that the above algorithm converges (in maybe an infinite number of steps), there exists a finite $l$ such that $\mathbb{K}_{l}^{\lambda, *}$ is $\hat{\lambda}$-contractive for any $\lambda<\hat{\lambda} \leq 1$, following identical argumentations to [2, Theorem 3.2]. Hence, if $l_{\text {MAX }}$ is reached, bisection on $\hat{\lambda}$ can be used to find the smallest $\hat{\lambda}$ such that $\mathbb{K}_{l_{M A X}^{\lambda, *}}$ is $\hat{\lambda}$-contractive, by testing if $\mathbb{K}_{l_{M A X}^{\lambda, *}} \subset Q^{*}\left(\hat{\lambda} \mathbb{K}_{l_{M A X}^{\lambda, *}}^{\lambda}\right)$.

From the above results, for any $\left(x_{0}, \theta_{0}\right) \in \mathbb{K}_{\infty}^{\lambda, *}$ there exits a causal $u_{0}=u_{0}\left(x_{0}, \theta_{0}\right)$ such that the successor $\left(x_{1}=A_{\theta_{0}} x_{0}+B_{\theta_{0}} u_{0}, \theta_{1}\right)$ is admissible for any possible successor mode $\theta_{1} \in S\left(\theta_{0}\right)$, i.e., $x_{1} \in \cap_{\theta_{1} \in S\left(\theta_{0}\right)} \lambda \mathbb{K}_{\infty}^{\lambda,\left[\theta_{1}\right]}$.

Now, the following set in the original (non-augmented) state state space can be defined:

\section{Definition 2}

$l$-step controlled safe set:

$$
\mathbb{S}_{l}^{\lambda}:=\cap_{i \in \mathcal{M}} \mathbb{K}_{l}^{\lambda,[i]}
$$

This set denotes the set of initial states for which there exists a control action (causal, dependent on current mode) that fulfils $\left(x_{k}, \theta_{k}\right) \in \lambda^{k} \Omega^{*}$ for $0 \leq k \leq l$ under any admissible sequence (irrespective of the starting mode $\theta_{0}$ ).

The name comes because starting in such state is "safe" (constraints not violated) at least for $l$ steps, even if $\theta_{0}$ is not yet known (assuming it will later be, of course).

After convergence, the sets $\mathbb{K}_{\infty}^{\lambda,[i]}$ are those for which an initial state in them, with initial mode $i$ can be kept in the admissible regions forever, and steered to the origin with decay-rate $\lambda$. Then, $\mathbb{S}_{\infty}^{\lambda}:=\cap_{i \in \mathcal{M}} \mathbb{K}_{\infty}^{\lambda,[i]}$ (to be named as maximal controlled safe set) denotes the set of states for which a causal admissible controller exists such that constraints will never be violated in the future, irrespective of the starting mode.

\section{Remark 2}

Note that the maximal safe set $\mathbb{S}_{\infty}^{\lambda}$ is, in general, not $\lambda$-contractive, in the sense of [4]. Indeed, the truly $\lambda$-contractive set is $\mathbb{K}_{\infty}^{\lambda, *}$; so, any state starting in $\mathbb{S}_{\infty}^{\lambda}$ will be kept at a future time $k$ inside $\cup_{i \in \mathcal{M}} \lambda^{k} \mathbb{K}_{\infty}^{\lambda,[i]}$. A particular case in which $\mathbb{S}_{\infty}^{\lambda}$ is $\lambda$-contractive is discussed below.

\section{Lemma 1}

Under arbitrary switching (i.e., $\pi_{j i} \neq 0$ for all $i, j$ ), the maximal controlled safe set $\mathbb{S}_{\infty}^{\lambda}$ is control $\lambda$-contractive. It is, actually, the maximal control $\lambda$-contractive set with mode-dependent control action.

\section{Proof}

Consider $\mathbb{K}_{\infty}^{\lambda, *}$ which is maximal and fulfils, due to convergence:

$$
\mathbb{K}_{\infty}^{\lambda, *}=Q^{*}\left(\mathbb{K}_{\infty}^{\lambda, *}\right)=\left\{\left(x_{0}, i\right) \in \Omega^{*}: \exists(u, i) \in \mathbb{U}^{*} \text { such that } A_{i} x_{0}+B_{i} u \in \lambda\left(\cap_{j \in \mathcal{M}} \mathbb{K}_{\infty}^{\lambda,[j]}\right)\right\},
$$


but, from (7), trivially (replacing the last intersection):

$$
\mathbb{K}_{\infty}^{\lambda, *}=Q^{*}\left(\mathbb{K}_{\infty}^{\lambda, *}\right)=\left\{\left(x_{0}, i\right) \in \Omega^{*}: \exists(u, i) \in \mathbb{U}^{*} \text { such that } A_{i} x_{0}+B_{i} u \in \lambda \mathbb{S}_{\infty}^{\lambda}\right\}
$$

Note that, if $x \in \mathbb{S}_{\infty}^{\lambda}$, then $(x, i) \in \mathbb{K}_{\infty}^{\lambda, *}$ for all $i$. Then, (9) entails that, whichever the mode, there exists a (mode-dependent) control action driving the successor state to the scaled set $\lambda \mathbb{S}_{\infty}^{\lambda}$. Thus, $\mathbb{S}_{\infty}^{\lambda}$ is control $\lambda$-contractive.

Note that, if $x \notin \mathbb{S}_{\infty}^{\lambda}$ then there exists $j$ such that $(x, j) \notin \mathbb{K}_{\infty}^{\lambda, *}$. Due to maximality of $\mathbb{K}_{\infty}^{\lambda, *}$, then there does not exist a control action keeping all the future states admissible; hence, $\mathbb{S}_{\infty}^{\lambda}$ is maximal, too.

\subsection{Sequence-dependent l-step sets}

The previous section enlarges the possible initial conditions with guaranteed convergence to the origin (with respect to the original robust sets in section 2.1). However, if the initial state and mode are not in $\mathbb{K}_{\infty}^{\lambda, *}$, then there exist a sequence of modes which will ultimately violate the constraints, whichever the controller. However, there might be some sequences for which a controller exists such that the state can be steered to $\mathbb{K}_{\infty}^{\lambda, *}$. So, there might be some probability of success in driving the state to the origin. This motivates the definition of the following $l$-step sets in the (non-augmented) state space for a particular sequence.

Given the system under study (1) and an arbitrary set $\mathcal{C}$, with associated mode-dependent state and input constraints, we will define the $l$-step sequence-set $Q_{\boldsymbol{\theta}}(\mathcal{C}), \boldsymbol{\theta} \in \mathcal{M}^{l}$ as:

$$
\begin{array}{r}
Q_{\boldsymbol{\theta}}(\mathcal{C}):=\left\{x_{0} \in \Omega^{\left[\theta_{0}\right]}: \exists \boldsymbol{u} \in \mathbb{U}_{\boldsymbol{\theta}} \text { such that }\left(x_{k}^{\boldsymbol{\theta}}\left(x_{0}, \boldsymbol{u}_{0: k-1}\right), \theta_{k}\right) \in \Omega^{*}\right. \\
\left.\forall k \in\{1,2, \ldots, l-1\}, x_{l}^{\boldsymbol{\theta}}\left(x_{0}, \boldsymbol{u}\right) \in \mathcal{C}\right\},
\end{array}
$$

where $x_{k}^{\boldsymbol{\theta}}$ is obtained with (5). Obviously, $Q_{\boldsymbol{\theta}}(\mathcal{C})$ is the set of initial states for which there exists and admissible control for sequence $\boldsymbol{\theta}$ such that the state is driven in an admissible trajectory to $\mathcal{C}$ in $\operatorname{len}(\boldsymbol{\theta})$ steps, while keeping the evolution of the state inside $\Omega^{*}$ for the first $l-1$ step.

When the sequence length is 1 , i.e., $\boldsymbol{\eta}=\{i\}, i \in \mathcal{M}$ the above expression will actually be a "classical" 1-step set, represented, with a slight abuse of notation, by:

$$
Q_{i}(\mathcal{C}):=\left\{x_{0} \in \Omega^{[i]}: \exists u_{0} \in \mathbb{U}^{[i]} \text { such that } A_{i} x_{0}+B_{i} u_{0} \in \mathcal{C}\right\}
$$

For instance, the one-step set in (6) could have been written as $Q^{*}\left(\mathcal{C}^{*}\right)=\left\{(x, i) \in \Omega^{*}: x \in\right.$ $\left.Q_{i}\left(\cap_{j \in S(i)} \mathcal{C}^{[j]}\right)\right\}$.

\section{Lemma 2}

Consider mode sequences $\boldsymbol{\eta} \in \mathcal{M}^{l_{1}}, \boldsymbol{\xi} \in \mathcal{M}^{l_{2}}, l_{1} \geq 1, l_{2} \geq 1$. Then, $Q_{\boldsymbol{\eta} \boldsymbol{\xi}}(\mathcal{C})=Q_{\boldsymbol{\eta}}\left(Q_{\boldsymbol{\xi}}(\mathcal{C})\right)$.

Proof (omitted) is straightforward from the definition. Such property will allow recursive definitions of the above sets, similar to standard algorithms.

\section{Corollary 1}

A particular case of the above lemma when $l_{1}=1$, i.e., $\boldsymbol{\eta}=\{i\}, i \in \mathcal{M}$ gives:

$$
Q_{i \xi}(\mathcal{C})=Q_{i}\left(Q_{\xi}(\mathcal{C})\right)
$$


As possible future mode sequences are infinite, there might be an infinite number of the above sequence-dependent sets. However, in some cases, the number of sets are finite and depending only in a few of the first sequence elements, motivating the lemma below.

Lemma 3

If $Q_{\boldsymbol{\eta} \xi_{1}}(\mathcal{C})=Q_{\boldsymbol{\eta}}(\mathcal{C})$ for all $\boldsymbol{\eta} \xi_{1} \in \overline{\mathcal{M}}^{l+1}$ then $Q_{\boldsymbol{\eta}}(\mathcal{C})=Q_{\boldsymbol{\eta}}(\mathcal{C})$ for any admissible sequence $\boldsymbol{\psi}$ of any arbitrary length.

Proof

Consider a sequence of length $l+2$ expressed as $\xi_{1} \in \mathcal{M}, \xi_{2} \in \mathcal{M}, \eta \in \overline{\mathcal{M}}^{l}$, such that $\eta \xi_{1} \xi_{2} \in$ $\overline{\mathcal{M}}^{l+2}$. Consider now the associated sequence set $Q_{\eta \xi_{1} \xi_{2}}$, Applying first Corollary 1, splitting out the first element of $\boldsymbol{\eta}$, i.e., $\boldsymbol{\eta} \xi_{1} \xi_{2}=\eta_{0} \boldsymbol{\eta}_{1: l-1} \xi_{1} \xi_{2}$, we have:

$$
Q_{\boldsymbol{\eta} \xi_{1} \xi_{2}}(\mathcal{C})=Q_{\eta_{0} \boldsymbol{\eta}_{1: l-1} \xi_{1} \xi_{2}}(\mathcal{C})=Q_{\eta_{0}}\left(Q_{\boldsymbol{\eta}_{1: l-1} \xi_{1} \xi_{2}}(\mathcal{C})\right)
$$

As $\boldsymbol{\eta}_{1: l-1} \xi_{1} \xi_{2} \in \overline{\mathcal{M}}^{l+1}, Q_{\boldsymbol{\eta}_{1: l-1} \xi_{1} \xi_{2}}=Q_{\boldsymbol{\eta}_{1: l-1} \xi_{1}}$ by assumption. Hence (13) can be expressed as:

$$
Q_{\eta_{0}}\left(Q_{\boldsymbol{\eta}_{1: l-1} \xi_{1} \xi_{2}}(\mathcal{C})\right)=Q_{\eta_{0}}\left(Q_{\boldsymbol{\eta}_{1: l-1} \xi_{1}}(\mathcal{C})\right)=Q_{\boldsymbol{\eta} \xi_{1}}(\mathcal{C})
$$

As $Q_{\boldsymbol{\eta} \xi_{1}}=Q_{\boldsymbol{\eta}}$ for all admissible $\boldsymbol{\eta} \xi_{1}$, (14) implies $Q_{\boldsymbol{\eta} \xi_{1} \xi_{2}}=Q_{\boldsymbol{\eta} \xi_{1}}=Q_{\boldsymbol{\eta}}$ for all admissible sequences. An induction argumentation makes $Q_{\eta \psi}=Q_{\eta}$ for any finite-length sequence $\psi$.

Recursive algorithm for $Q_{\eta}$. The finite collection of sets arising from the above lemma can be computed by Algorithm 2 (if it succeeds), with a target set $\mathcal{C}$ used to "seed" the recursions.

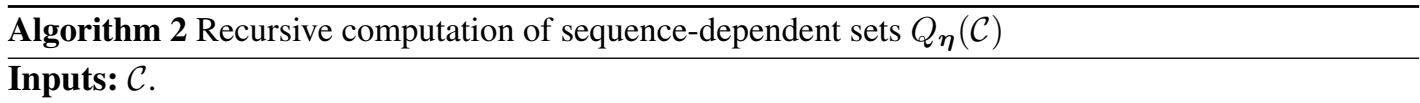

1. Start with all $\psi \in \mathcal{M}$. Compute $Q_{\psi}(\mathcal{C})$. If all $Q_{\psi}=\mathcal{C}$, let success=true; STOP.

2. Let $l=1$.

3. For all $\xi \in \mathcal{M}, \boldsymbol{\eta} \in \overline{\mathcal{M}}^{l}$ such that $\xi \boldsymbol{\eta}$ is admissible, compute $Q_{\xi \boldsymbol{\eta}}(\mathcal{C})$ using the one-step set in Lemma 2. $Q_{\xi \boldsymbol{\eta}}(\mathcal{C})=Q_{\xi}\left(Q_{\boldsymbol{\eta}}(\mathcal{C})\right)$.

4. If $Q_{\boldsymbol{\eta} \psi}(\mathcal{C})=Q_{\boldsymbol{\eta}}(\mathcal{C})$ for all $\psi \in \mathcal{M}, \boldsymbol{\eta} \in \overline{\mathcal{M}}^{l}$ such that $\psi \boldsymbol{\eta}$ is admissible, then let success $=$ true; $l_{c}=l$; STOP.

5. Let $l=l+1$, if $l<l_{\text {MAX }}$ go to step 2 else, let success=false; $l_{c}=l_{\text {MAX }}$; STOP.

If "success" ends up having a true value, the result is a list of converged sets $Q_{\boldsymbol{\eta}}(\mathcal{C})$, covering all $\boldsymbol{\eta} \in \overline{\mathcal{M}}^{l_{c}}$. Also, intermediate sets $Q_{\boldsymbol{\eta}}(\mathcal{C})$ with $\operatorname{len}(\boldsymbol{\eta})<l_{c}$ should also be stored for further interpretation, see Section 4. IF "success" were not true, the algorithm wouldn't have found the final converged sets; anyway, they can still be useful in some cases, as discussed in Section 4.3.

The interpretation of the converged set $Q_{\eta}=Q_{\eta \xi}$ is that for all $x$ in $Q_{\eta}$ there exists a control action driving the state to $\mathcal{C}$ under any sequence starting with $\boldsymbol{\eta}$. However, note that the control action might be sequence-dependent, so it would need a "non-causal" controller. Hence, further developments are needed in order to devise causal controllers, as discussed next. 


\section{RELIABLE ON-LINE CONTROLLER DESIGN}

The above sequence-dependent sets can be used in order to decide a course of action to reach the origin with high likelihood. Let us consider each of the two mode information set-ups, i.e., controller knows current mode or not.

\subsection{Controller with known current mode in on-line operation}

Of course, if at a particular instant, say $t=0$, we have $\left(x_{0}, \theta_{0}\right) \in \mathbb{K}_{\infty}^{\lambda, *}$, there exists a control law keeping the next state and mode in $\mathbb{K}_{\infty}^{\lambda, *}$, so that this controller is the one to be applied ensuring $100 \%$ likelihood of being admissible at all future times.

When $\left(x_{0}, \theta_{0}\right) \notin \mathbb{K}_{\infty}^{\lambda, *}$, the following corollary combines the two above algorithms by using the results of Algorithm 1 as seeds $\mathcal{C}$ of Algorithm 2.

Corollary 2

Given $\gamma \in \overline{\mathcal{M}}^{l+1}, \gamma=\boldsymbol{\eta} \psi$, for all $x_{0} \in Q_{\boldsymbol{\eta}}\left(\mathbb{K}_{\infty}^{\lambda,[\psi]}\right)$, there exists a control law driving the state to $\mathbb{K}_{\infty}^{\lambda, *}$ from $x_{0}$ under sequence $\boldsymbol{\eta}$, and remaining in $\mathbb{K}_{\infty}^{\lambda, *}$ thereafter for any subsequent mode sequence beginning with $\psi$, converging to the origin with contraction rate $\lambda$.

Proof

The proof is trivial from the definition of $Q_{\boldsymbol{\eta}}\left(\mathbb{K}_{\infty}^{\lambda,[\psi]}\right)$, the $\lambda$-contractiveness of $\mathbb{K}_{\infty}^{\lambda, *}$ and the fact that $(x, \psi) \in \mathbb{K}_{\infty}^{\lambda, *}$ for any $x \in \mathbb{K}_{\infty}^{\lambda,[\psi]}$.

Note that Corollary 2 does not require convergence of Algorithm 2, but only convergence of Algorithm 1. Note, too, that the sets $Q_{\boldsymbol{\eta}}\left(\mathbb{K}_{\infty}^{\lambda,[\psi]}\right)$ get "larger" as len $(\boldsymbol{\eta})$ increases. In precise terms:

Corollary 3

Given $\gamma \in \overline{\mathcal{M}}^{l}, \gamma=\boldsymbol{\eta} \psi_{1} \psi_{2}$, then $Q_{\boldsymbol{\eta}}\left(\mathbb{K}_{\infty}^{\lambda,\left[\psi_{1}\right]}\right) \subset Q_{\boldsymbol{\eta} \psi_{1}}\left(\mathbb{K}_{\infty}^{\lambda,\left[\psi_{2}\right]}\right)$.

Proof

Indeed, the set of $x_{0}$ which can be driven to the origin with any arbitrary sequence starting with $\boldsymbol{\eta} \psi_{1}$ -i.e, $Q_{\boldsymbol{\eta}}\left(\mathbb{K}_{\infty}^{\lambda,\left[\psi_{1}\right]}\right)$ from Corollary $2-$ is a subset of those which can be driven with the particular case $\boldsymbol{\eta} \psi_{1} \psi_{2}$-i.e., $Q_{\boldsymbol{\eta} \psi_{1}}\left(\mathbb{K}_{\infty}^{\lambda,\left[\psi_{2}\right]}\right)-$.

So, as Algorithm 2 progresses, a larger family of nested sets (in the above sense) is obtained, until convergence (if it happens to occur) or exhaustion of computational resources. A practical interpretation of the above corollary means that progressively longer sequences will reach points further away from the origin. As such sequences will be less likely, initial conditions far away from the origin will have lower likelihood of being steered to the origin than those close to it, as intuitively expected.

4.1.1. Reliable control algorithm. The above results can be used in on-line control to obtain a control action which drives the state to the maximal set $\mathbb{K}_{\infty}^{\lambda, *}$ with a given probability (associated to the most likely sequence).

As above discussed, if $\left(x_{0}, \theta_{0}\right) \in \mathbb{K}_{\infty}^{\lambda, *}, 100 \%$ reliability can be guaranteed. Otherwise, the control action resulting from Algorithm 3 below can be easily computed (at least when all involved sets are 
polytopic ones: everything amounts to linear constraints). Let us first present the algorithm, and discuss later the meaning of the involved steps.

Algorithm inputs are current state, current mode and all the sets resulting from Algorithm 2, either until convergence or until $l_{c}=l_{M A X}$ has been reached without success. As $l_{c}$ can be different for each $\mathbb{K}_{\infty}^{\lambda,[\xi]}, \xi \in \mathcal{M}$, when Algorithm 2 is run, it will either converge to a finite $l_{c, \xi}$ or reach $l_{c, \xi}=l_{\max }$ without success.

Algorithm 3 Reliable On-line Controller

Inputs: $x_{0}, \theta_{0}, Q_{\boldsymbol{\eta}}\left(\mathbb{K}_{\infty}^{\lambda,[\xi]}\right), \boldsymbol{\eta} \xi \in \overline{\mathcal{M}}^{l_{c, \xi}}$

1. If $x_{0} \in \mathbb{K}_{\infty}^{\lambda,\left[\theta_{0}\right]}$, compute any control such that $x_{1} \in \cap_{j \in S\left(\theta_{0}\right)} \mathbb{K}_{\infty}^{\lambda,[j]}$. EXIT.

2. Else, compute the set of sequences

$$
\begin{aligned}
\boldsymbol{\Gamma}\left(x_{0}, \theta_{0}\right):=\left\{\boldsymbol{\gamma}: \boldsymbol{\gamma}=\boldsymbol{\eta} \xi, 1 \leq \operatorname{len}(\boldsymbol{\eta}) \leq l_{c, \xi}, \xi \in \mathcal{M}, x_{0} \in Q_{\boldsymbol{\eta}}\left(\mathbb{K}_{\infty}^{\lambda,[\xi]}\right)\right. \\
\left.\boldsymbol{\gamma} \text { is admissible, } \gamma_{0}=\theta_{0}\right\}
\end{aligned}
$$

3. if $\boldsymbol{\Gamma}\left(x_{0}, \theta_{0}\right)=\emptyset$, Throw("Failure"); ExIT.

4. Else, determine the sequence $\boldsymbol{\beta}$ in $\boldsymbol{\Gamma}\left(x_{0}, \theta_{0}\right)$ with highest probability (conditioned to $\gamma_{0}=\theta_{0}$ ), i.e.,

$$
\boldsymbol{\beta}:=\arg \max _{\boldsymbol{\gamma} \in \boldsymbol{\Gamma}\left(x_{0}, \theta_{0}\right)} \operatorname{Pr}\left(\boldsymbol{\gamma} \mid \gamma_{0}=\theta_{0}\right)
$$

5. Let $h:=\operatorname{len}(\boldsymbol{\beta})$. Compute a control steering the next state to $Q_{\boldsymbol{\beta}_{1: h-2}}\left(\mathbb{K}_{\infty}^{\lambda,[\xi]}\right)$, being $\xi=$ $\boldsymbol{\beta}_{h-1}$, i.e., the last element of $\boldsymbol{\beta}$. EXIT.

Depending on the initial state, the presented algorithm can succeed in obtaining a stabilizing controller or not. Let us discuss such situations, detected at Step 3.

\section{Interpretation of Step 3.}

\section{Proposition 1}

If $\boldsymbol{\Gamma}\left(x_{0}, \theta_{0}\right)=\emptyset$, and $l_{c, \xi}<l_{M A X}$ for all $\xi$, then there does not exist a control law which drives the state to the origin, irrespective of the mode sequence: the probability of success is zero.

Proof

Indeed, there is no $Q_{\boldsymbol{\eta}}\left(\mathbb{K}_{\infty}^{\lambda,[\xi]}\right)$ with $\boldsymbol{\eta}$ starting with $\theta_{0}$ to which $x_{0}$ belongs, so, as Algorithm 2 converged, there does not exist any arbitrary-length sequence for which there exists a control sequence bringing it to $\mathbb{K}_{\infty}^{\lambda, *}$.

The statement Throw ( 'Failure' ') indicates that the situation in Proposition 1 did occur and, hence, there is no possibility of driving the state to the origin violating constraints: suitable abort or constraint relaxation procedures should be handled elsewhere.

\section{Remark 3}

Actually, in the algorithm implementation, if $l_{c, \xi}=l_{M A X}$ for some $\xi$ (i.e., non-convergence of the prior Algorithm 2 for at least one $\mathbb{K}_{\infty}^{\lambda,[\xi]}$ ) the meaning would be slightly different: the allowed computational resources would not prove existence of a stabilizing controller for the explored sequences; however, there might be longer ones for which such controller exists. Again, backup controllers for such a case are out of the scope of this work. 
Interpretation of steps 4 and 5 (success). Regarding steps 4 and 5 , note that if $\boldsymbol{\Gamma}\left(x_{0}, \theta_{0}\right) \neq \emptyset$, a controller which steers the state to the origin for any sequence in $\boldsymbol{\Gamma}\left(x_{0}, \theta_{0}\right)$ exists. However, the control action might be different for each sequence, of course; then, as the controller must be causal, betting on the most probable one for the future is the choice proposed in Algorithm 3.

Indeed, being $h=\operatorname{len}(\boldsymbol{\beta})$, consider any controller which steers the next state to the "successor set" $Q_{\boldsymbol{\beta}_{1: h-2}}\left(\mathbb{K}_{\infty}^{\lambda,[\xi]}\right)$, being $\xi=\boldsymbol{\beta}_{h-1}$, i.e., $\xi$ is the last element of $\boldsymbol{\beta}$. By definition, such controller does exist and its computation requires just a one-step feasibility condition, trivial to solve for in the linear case.

Note also that, as sequence-dependent sets are nested, in the sense precised in Corollary 3, if $\boldsymbol{\gamma} \in \boldsymbol{\Gamma}\left(x_{0}, \gamma_{0}\right)$ then any longer sequences starting with $\boldsymbol{\gamma}$ also belong to $\boldsymbol{\Gamma}\left(x_{0}, \gamma_{0}\right)$; however, their probability will be, evidently, lower. To avoid searching for irrelevant sequences, we will denote as $\operatorname{Trim}(\boldsymbol{\Gamma})$ the result of removing from a set of sequences $\boldsymbol{\Gamma}$ any sequence whose starting subsequence is already in $\boldsymbol{\Gamma}$. Obviously, $\boldsymbol{\Gamma}$ should actually be replaced, then, by $\operatorname{Trim}(\boldsymbol{\Gamma})$ in (16).

Off-line reliability computation. If the initial mode is not known at the time of reliability determination (i.e., off-line, prior to actual operation), then $100 \%$ reliability can be guaranteed only for $x_{0} \in \mathbb{S}_{\infty}^{\lambda}$. Otherwise, i.e., considering a particular $x_{0} \notin \mathbb{S}_{\infty}^{\lambda}$, let us assume that only an a priori probability vector for each mode $p=\left\{p_{1}, \ldots, p_{M}\right\}$ with $p_{i}:=\operatorname{Pr}\left(\theta_{0}=i\right), i \in \mathcal{M}$ is available.

To incorporate the concepts in Step 1 without knowledge of initial mode, instead of (15) we must consider:

$$
\boldsymbol{\Gamma}_{2}\left(x_{0}\right):=\left\{\boldsymbol{\gamma}: \boldsymbol{\gamma}=\boldsymbol{\eta} \xi, 0 \leq \operatorname{len}(\boldsymbol{\eta}) \leq l_{c, \xi}, \xi \in \mathcal{M}, x_{0} \in Q_{\boldsymbol{\eta}}\left(\mathbb{K}_{\infty}^{\lambda,[\xi]}\right), \boldsymbol{\gamma} \text { is admissible }\right\}
$$

abusing the notation by understanding $Q_{\boldsymbol{\eta}}\left(\mathbb{K}_{\infty}^{\lambda,[\xi]}\right):=\mathbb{K}_{\infty}^{\lambda,[\xi]}$ for zero-length $\boldsymbol{\eta}$.

Analogously to Proposition 1, if $\boldsymbol{\Gamma}_{2}\left(x_{0}\right)=\emptyset$ and $l_{c, \xi}<l_{M A X}$ for all $\xi \in \mathcal{M}$ (convergence) there is no sequence for any initial mode for which the state could be steered to the origin. Otherwise, a controller which steers the state to the origin for some sequences exists. A lower bound for the probability of success of the above algorithm can be obtained with the result below:

\section{Lemma 4}

The controller in Algorithm 3 has a probability of driving the state to the origin ${ }^{\dagger}$ of, at least:

$$
\underline{p}:=\sum_{i \in \mathcal{M}}\left(p_{i} \cdot \max _{\gamma \in \operatorname{Trim}\left(\boldsymbol{\Gamma}_{2}\left(x_{0}\right)\right)} \operatorname{Pr}\left(\gamma \mid \gamma_{0}=i\right)\right)
$$

Proof

Indeed, the sequence $\boldsymbol{\beta}_{2}\left(x_{0}, i\right)$ from $\boldsymbol{\Gamma}_{2}\left(x_{0}\right)$, given by

$$
\boldsymbol{\beta}_{2}\left(x_{0}, i\right):=\arg \max _{\boldsymbol{\gamma} \in \boldsymbol{\Gamma}_{2}\left(x_{0}\right)} \operatorname{Pr}\left(\boldsymbol{\gamma} \mid \gamma_{0}=i\right) \quad i \in \mathcal{M}
$$

would be the sequence $\boldsymbol{\beta}$ that Algorithm 3 would produce if $\theta_{0}=i$ at the moment of computing the control action. It is straightforward to see that summation of the probability of each of the

${ }_{\dagger}^{\dagger}$ actually, with geometric contraction rate $\lambda$ after $h$ steps 
$\boldsymbol{\beta}_{2}\left(x_{0}, i\right)$ conditioned to its starting element, multiplied by the probability of each starting mode gives (18).

\subsection{Controller with no on-line mode information}

If the current mode is not available to the controller, a controller can be crafted using as seed to Algorithm 2 the "classical" maximal $\lambda$-contractive controllable set from Section (2.1), i.e., using $\mathcal{K}_{\infty}^{\lambda}$ in Step 1 (instead of the previously used augmented $\mathbb{K}_{\infty}^{\lambda, *}$ ). Also, (15) in the controller algorithm must be redefined, changing $\Gamma$ to:

$$
\Gamma_{3}\left(x_{0}\right):=\left\{\gamma: 1 \leq \operatorname{len}(\gamma) \leq l, x_{0} \in Q_{\gamma}\left(\mathcal{K}_{\infty}^{\lambda}\right), \gamma \text { is admissible }\right\}
$$

and (16) should be changed to finding the sequence with maximal probability in (20):

$$
\boldsymbol{\beta}:=\arg \max _{\boldsymbol{\gamma} \in \operatorname{Trim}\left(\boldsymbol{\Gamma}_{3}\left(x_{0}\right)\right)} \operatorname{Pr}(\boldsymbol{\gamma})
$$

where probability $\operatorname{Pr}(\gamma)$ is, obviously, computed using whatever prior information $p$ available (or, for instance, assuming stationary state of the Markov chain).

Then, the control law steering the state to the successor $\operatorname{set} Q_{\gamma(1: \text { end })}\left(\mathcal{K}_{\infty}^{\lambda}\right)$ has at least probability $\underline{p}=\operatorname{prob}\left(\boldsymbol{\beta}\left(x_{0}\right)\right)$ of success ${ }^{\ddagger}$. Further details are omitted as they are almost identical to the knownmode case. Obviously, the resulting controller has lower reliability than the one in Section 4.1.

\subsection{Discussion and computational issues}

Obviously, the applicability of the presented results is limited by the required computational load for the proposed algorithms. In the polyhedral case, computational issues arise because of two reasons:

- on one hand, due to the possible increase of the number of vertices of the $M$ sets which get iteratively refined in Algorithm 1: the time taken to compute each step increases with $l$ (due to a larger number of vertices in the involved polytopes),

- on the other hand, because of the heavy increase of the number of sequences in Algorithm 2.

The computational burden of Algorithm 1 is well studied, as it is a higher-dimensional version of standard control-invariant set algorithms; such algorithms and their computational issues are described in [4, 7] and references therein: although the number of involved sets in Algorithm 1 does not increase with the number of iterations, the basic drawback lies in vertex enumeration/projection steps whose computational demands make them, in particular, very hard to solve for systems with a large number of input variables.

Regarding the sequence-dependent sets in Algorithm 2, the number of possible sequences to consider grows exponentially with the number of steps $l$ in most cases (for instance, for all regular Markov chains). In particular, it can be easily proved that the number of sets for a given value of $l$ (in the $M$ needed runs of Algorithm 2) is given by the sum of the elements of the $l$-th power of

\footnotetext{
‡Indeed, the summation in (18) cannot be made because the controller will not be able to make the choice between the different $\boldsymbol{\beta}\left(x_{0}, i\right)$ that (18) assumes, as mode $i$ will not be known. Hence, the proposed option in (21) is betting for the (single) most likely sequence driving to the robust $\lambda$-contractive controllable set. Note that, due to the lack of mode information, the probability of $\boldsymbol{\beta}$ in (21) is lower than the bound $p$ in (18).
} 
the incidence matrix $\mathcal{I}^{l}$. So, denoting by $\bar{\lambda}$ the largest eigenvalue of $\mathcal{I}$, for long enough sequences, the number of sequence-dependent sets may increase with $\mathcal{O}\left(\bar{\lambda}^{l}\right)$. Note however, that some of the sets obtained with (12) might be coincident with previously-computed ones so further one-steps sets from them can be skipped -in fact, when such thing happens with all sets, we have the conditions in Lemma 3-.

A last issue is the existence of multiple control actions steering the state to the successor set: the on-line control action can be computed, for instance, minimizing a suitable cost index, say, one-step control effort, if the solution were not unique (using linear or quadratic programming).

Algorithm modifications. From the above discussion, there might be not enough computational resources to achieve full convergence of Algorithm 2 (or the algorithm might not converge in a finite number of iterations). When not converged, if initial state is outside all of the obtained sets on-line finite-horizon controllers might be conceived trying to reach (or land "close enough" to) some of the computed sets.

A second modification can be suggested if Algorithm 1 does not converge: in such a case, Algorithm 2 can be seeded with any subset of either $\mathcal{K}_{\infty}^{\lambda}$ (unknown mode) or $\mathbb{K}_{\infty}^{\lambda, *}$ (known mode), computed, for instance, stopping Algorithm 1 when a contractive set is found (Remark 1), or via Lyapunov functions and LMIs [20, 21] or, plainly, the origin (safe, for sure, even for unstable plants).

Also, alternative controllers in step 5 of Algorithm 3 could be crafted, trying to steer the state to the intersection of several of the $Q_{\eta}$ sets arising from $\Gamma$, if possible. In that way, the controller would "bet" on several sequences -at least for one sample-. Finding common control actions for several sequences in multi-step settings might also be addressable. However, the number of possible sequence groups is $2^{q}-1$ being $q$ the number of elements of $\boldsymbol{\Gamma}$; also, projections involved in computing the controllable sets severely hinder computation of multi-step feasible sets. Hence, the computational complexity of determining the maximum-reliability controller and its associated controllable sets appears to be insurmountable except for simplistic cases.

As a last remark, if transition probabilities (or prior information) were uncertain, the bound $p$ should be computed maximizing the worst-case probability estimate. Anyway, as the shape of sequence-dependent sets is independent of the probability computation, the "uncertain probability" case does not modify any essential aspect of the procedures presented in this manuscript so details are left to the reader.

\section{NUMERICAL EXAMPLE}

Consider the second-order MJLS (1) with three operating modes, $i=\{1,2,3\}$. The corresponding parameters are given in Table I. Also, a geometric contraction rate specification $\lambda=0.98$ was chosen. For off-line reliability computations, the prior probability of each mode $\operatorname{Pr}\left(\theta_{0}=i\right)$ has been computed assuming stationary state of the mode Markov chain, resulting in $p_{1}=0.7353$, $p_{2}=0.0294$ and $p_{3}=0.2353$.

First, the sets $\mathbb{K}_{\infty}^{\lambda,[i]}$ are computed by executing Algorithm 1 until convergence using the one-step set (11). This initial algorithm converged in four steps. The maximal safe set $\mathbb{S}_{\infty}^{\lambda}$ is determined, according to Definition 2. So, this set has probability 1 of success. 
Then, Algorithm (2) is run three times, setting for each execution, the corresponding $\mathbb{K}_{\infty}^{\lambda,[i]}$ as seed. The result is a list of sets, i.e., $Q_{\boldsymbol{\eta}}\left(\mathbb{K}_{\infty}^{\lambda,[1]}\right), Q_{\boldsymbol{\xi}}\left(\mathbb{K}_{\infty}^{\lambda,[2]}\right)$ and $Q_{\boldsymbol{\phi}}\left(\mathbb{K}_{\infty}^{\lambda,[3]}\right)$. The converged lengths are, too, $l_{c, 1}=l_{c, 2}=l_{c, 3}=4$. The number of possible sequences, from the powers of the incidence matrix $\mathcal{I}$, are, for $l$ ranging from zero (the 3 maximal sets $\left.\mathbb{K}_{\infty}^{\lambda,[1]}\right)$ ) to 4 is $\{3,7,17,41,99\}$. However, the number of new sequence-dependent sets appearing at each step (i.e., different from those sets present in previous steps, with default MPT tolerances) is only $\{3,6,11,3,0\}$, so we have after convergence (Lemma 3 ) a total of 23 sets. The new sets at each step appear in Figure 1.

Using the 23 obtained sets, their projection on $x$-space would lead to Figure 2; however, in the figure, a color map has been superimposed representing the reliability bound $p$ from (18) (both numerical figures and yellow (high reliability) to green (low reliability) fillings appear). Regions for which no stabilizing controller (fulfilling the required constraints) exists for any sequence are filled in white. The set $\mathbb{S}_{\infty}^{\lambda}$ is, obviously, labelled with reliability bound equal to one in the figure.

In order to detail the probability computation, consider, for instance, an arbitrarily chosen point $x_{0}=[3.433 ; 7.157]$, which does not belong to $\mathbb{S}_{\infty}^{\lambda}$. However, $x_{0}$ belongs to (trimming longer sequences -Corollary 3-):

$$
\begin{aligned}
& Q_{\boldsymbol{\eta}}\left(\mathbb{K}_{\infty}^{\lambda,[1]}\right) \quad \text { for } \boldsymbol{\eta} \in\{[1\},\{1,2\},\{3,2\}\} \\
& Q_{\boldsymbol{\xi}}\left(\mathbb{K}_{\infty}^{\lambda,[2]}\right) \quad \text { for } \boldsymbol{\xi} \in\{\{1\},\{3\},\{1,1\},\{3,3\},\{1,1,1\}\} \\
& Q_{\boldsymbol{\phi}}\left(\mathbb{K}_{\infty}^{\lambda,[3]}\right) \text { for } \boldsymbol{\phi} \in\{\{1,2\},\{1,1,2\}\} \\
& \quad \mathbb{K}_{\infty}^{\lambda,[3]}
\end{aligned}
$$

Let us consider an on-line operation measuring $\theta_{0}=1$. Then, the most likely sequence $\gamma$ in $\boldsymbol{\Gamma}\left(x_{0}, 1\right)$ from (15), i.e., conditioned to initial mode equal to 1 , is $\{1,1\}$ (probability 0.98 , from the boxed $\boldsymbol{\eta}$ in (22) above). Then, a control action steering the successor state to $\mathbb{K}_{\infty}^{\lambda,[1]}$ would be proposed by Algorithm 3.

For off-line reliability computation (a priori), apart from the case $\theta_{0}=1$, the other remaining two possibilities should be considered. For $\theta_{0}=2$, there does not exist any sequence in $\boldsymbol{\Gamma}_{2}\left(x_{0}\right)$, so the system will violate constraints at some point in the future, for sure. For $\theta_{0}=3$, as $x_{0}$ belongs to

\begin{tabular}{|c|c|c|c|}
\hline \multirow{2}{*}{ Parameters } & \multicolumn{3}{|c|}{ Operation modes } \\
\hline & $i=1$ & $i=2$ & $i=3$ \\
\hline$A_{i}$ & $\begin{array}{cc}-0.8891 & -0.8149 \\
0.8149 & -0.8891\end{array}$ & $\begin{array}{cc}-0.4446 & 0.4074 \\
-0.4074 & -0.4446\end{array}$ & $\begin{array}{cc}0.5636 & -0.7240 \\
0.7240 & 0.5636\end{array}$ \\
\hline$B_{i}$ & {$\left[\begin{array}{l}0 \\
1\end{array}\right]$} & {$\left[\begin{array}{c}0 \\
0.5\end{array}\right]$} & $\left.\begin{array}{c}0 \\
1.2\end{array}\right]$ \\
\hline$\Omega^{[i]}$ & {$\left[\begin{array}{l}-10 \leq x_{1} \leq 10 \\
-10 \leq x_{2} \leq 10\end{array}\right.$} & {$\left[\begin{array}{rl}-5 & \leq x_{1} \leq 5 \\
-12 & \leq x_{2} \leq 12\end{array}\right.$} & $\begin{array}{c}-5 \leq x_{1} \leq 5 \\
-14 \leq x_{2} \leq 8\end{array}$ \\
\hline $\mathbb{U}=$ & $\leq u \leq 9]$ & $\left.\begin{array}{rrc}0.98 & 0.5 & 0 \\
0.02 & 0.1 & 0.05 \\
0 & 0.4 & 0.95\end{array}\right]$, & $\mathcal{I}=\left[\begin{array}{lll}1 & 1 & 0 \\
1 & 1 & 1 \\
0 & 1 & 1\end{array}\right]$ \\
\hline
\end{tabular}
$\mathbb{K}_{\infty}^{\lambda,[3]}$, it has probability one of success if initial mode were 3 . Therefore, its probability bound $\underline{p}$ is $0.7353 * 0.98+0.2353 * 1=0.9559$.

Table I. Example parameters. 


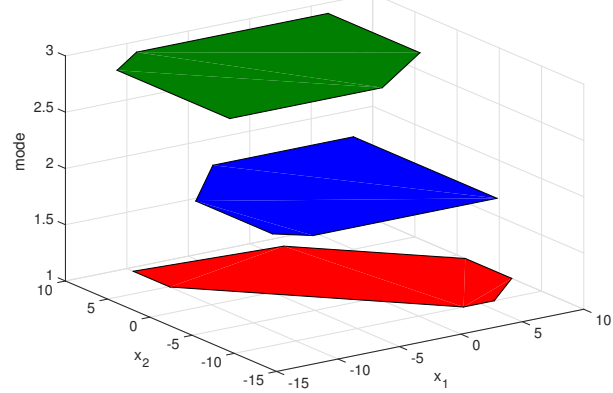

(a)

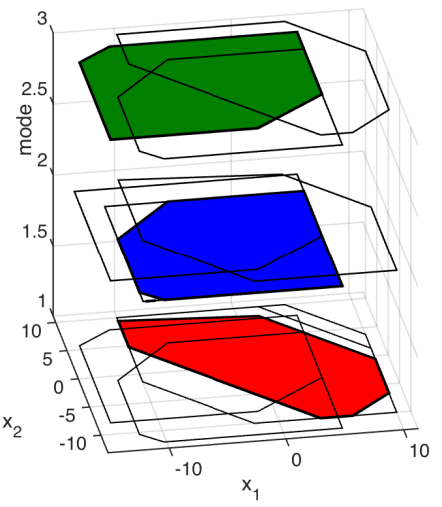

(c)

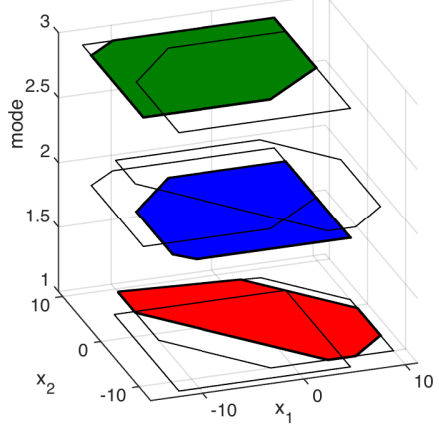

(b)

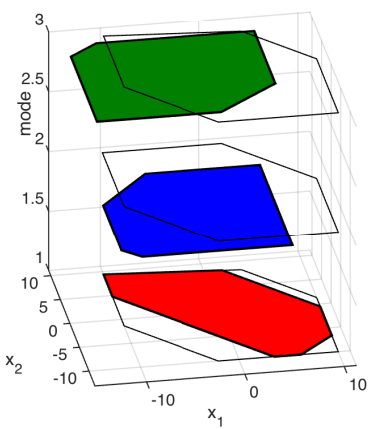

(d)

Figure 1. (a) Maximal controllable sets for each mode. [The projection of these sets onto the $x$-space are the dashed lines in Figure 3];

(b) 1-step sets from each of the sets in plot (a), for each mode (six new sets appearing);

(c) 2-step sets (11 new sets appearing);

(d) 3-step sets (three new sets appearing).

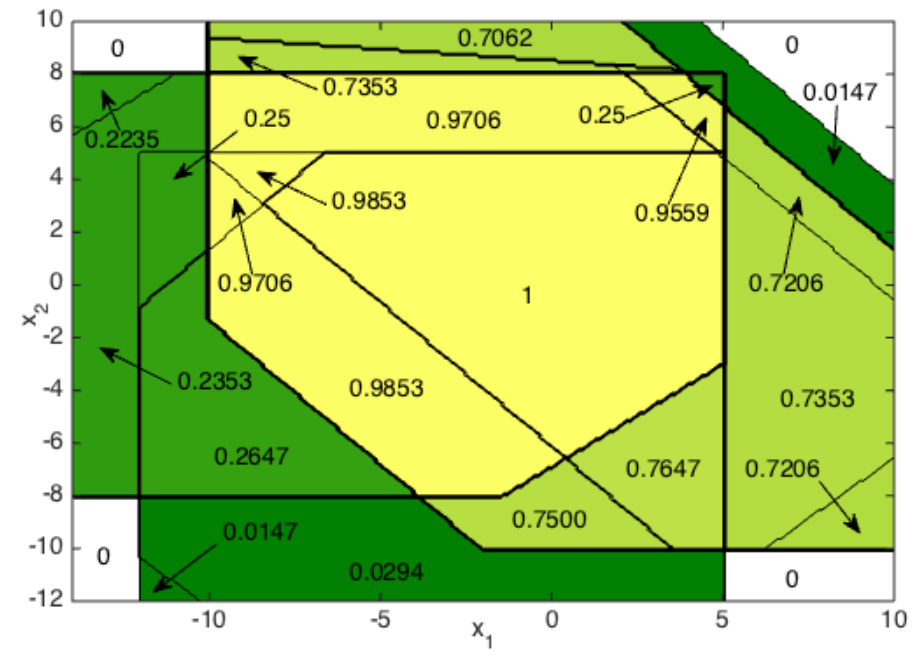

Figure 2. Reliability bound $\mathrm{p}$ for different initial states, under stationary mode assumption. 


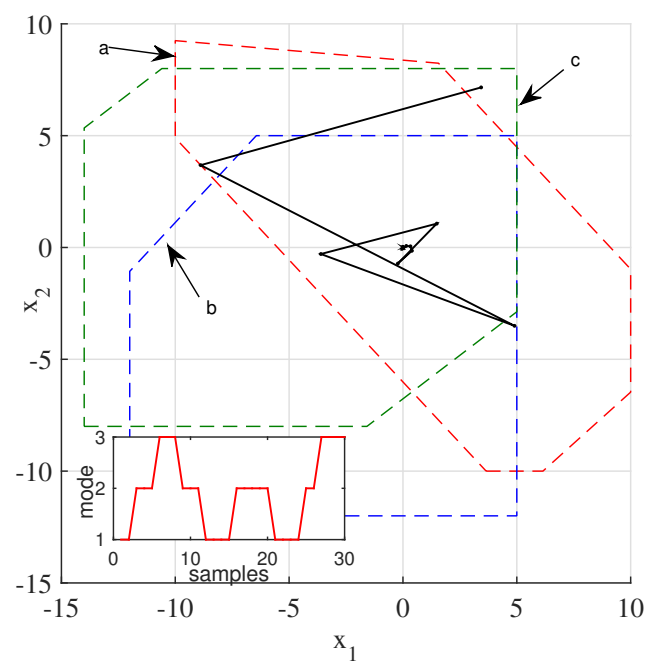

Figure 3. (Solid black line) state response of the closed-loop system under mode evolution $\theta$ indicated in the bottom-left sub-plot. Dashed lines depict the sets from Algorithm 1: a) $\mathbb{K}_{\infty}^{\lambda,[1]}$, b) $\mathbb{K}_{\infty}^{\lambda,[2]}$ and c) $\mathbb{K}_{\infty}^{\lambda,[3]}$.

In the same way as done with $x_{0}=[3.433 ; 7.157]$, analogous computations can be carried out for each of the depicted regions in Figure 2.

Closed-loop simulation. Figure 3 depicts a simulation of the time response of the above chosen point $x_{0}$ under sequence $\boldsymbol{\eta}=\{1,1,2,2,2,3,3,3,2,2, \ldots\}$ and the controller in Algorithm 3, choosing, if solution were non-unique, the one minimizing $u_{k}^{2}$. The three sets conforming $\mathbb{K}_{\infty}^{\lambda, *}$ from Algorithm 1, i.e., $\mathbb{K}_{\infty}^{\lambda,[1]}, \mathbb{K}_{\infty}^{\lambda,[2]}$ and $\mathbb{K}_{\infty}^{\lambda,[3]}$ have also been plotted there. As discussed above, as $x_{0} \in Q_{1}\left(\mathbb{K}_{\infty}^{\lambda,[1]}\right)$, the system reaches $\mathbb{K}_{\infty}^{\lambda, *}$ in one step, as expected.

\section{CONCLUSIONS}

This work has presented algorithms to generate a sequence of nested sets and control actions associated to them for reliable control. The proposed controllers using sequence-dependent sets have a guaranteed minimum reliability bound, understood as likelihood of driving the state to the origin without violating constraints. The basic idea is the fact that, as soon as the state lands at particular "safe" sets, reliability is $100 \%$. Then, some probability of success $\underline{p}$ can be asserted for initial conditions in the (larger) sets for which there exists a finite-length sequence with a controller steering the state to such safe sets.

The proposed sets are a probabilistic generalization of the maximal robust $\lambda$-contractive sets in prior literature. Mode-dependent constraints are incorporated, too. As intuitively expected, the further away initial conditions are from the origin, the lower the computed (state-dependent) reliability bounds are. 


\section{ACKNOWLEDGEMENTS}

This work has been supported by projects DPI2011-27845-C02-01, DPI2011-27845-C02-02, and FPU grant FPU12/02107, both from Spanish Government.

\section{REFERENCES}

1. Gilbert EG, Tan KT. Linear systems with state and control constraints: The theory and application of maximal output admissible sets. Automatic Control, IEEE Transactions on 1991; 36(9):1008-1020.

2. Blanchini F. Ultimate boundedness control for uncertain discrete-time systems via set-induced lyapunov functions. Automatic Control, IEEE Transactions on 1994; 39(2):428-433.

3. Blanchini F. Set invariance in control. Automatica 1999; 35(11):1747-1767.

4. Kerrigan EC. Robust constraint satisfaction: Invariant sets and predictive control. PhD Thesis, PhD thesis, Cambridge 2000.

5. Ariño C, Pérez E, Sala A, Bedate F. Polytopic invariant and contractive sets for closed-loop discrete fuzzy systems. Journal of the Franklin Institute 2014; 351(7):3559-3576.

6. Dehghan M, Ong CJ. Discrete-time switching linear system with constraints: Characterization and computation of invariant sets under dwell-time consideration. Automatica 2012; 48(5):964-969.

7. Kvasnica M, Grieder P, Baotić M, Morari M. Multi-parametric toolbox (mpt). Hybrid systems: computation and control. Springer, 2004; 448-462.

8. Herceg M, Kvasnica M, Jones CN, Morari M. Multi-parametric toolbox 3.0. Control Conference (ECC), 2013 European, IEEE, 2013; 502-510.

9. do Valle Costa OL, Fragoso MMD, Marques RP. Discrete time Markov jump linear systems. Springer, 2005.

10. Zhang L, Shi Y, Chen T, Huang B. A new method for stabilization of networked control systems with random delays. Automatic Control, IEEE Transactions on 2005; 50(8):1177-1181.

11. Shi Y, Yu B. Output feedback stabilization of networked control systems with random delays modeled by markov chains. Automatic Control, IEEE Transactions on 2009; 54(7):1668-1674.

12. Li H, Gao H, Shi P, Zhao X. Fault-tolerant control of markovian jump stochastic systems via the augmented sliding mode observer approach. Automatica 2014; 50(7):1825-1834.

13. Liu M, Shi P, Zhang L, Zhao X. Fault-tolerant control for nonlinear markovian jump systems via proportional and derivative sliding mode observer technique. Circuits and Systems I: Regular Papers, IEEE Transactions on 2011; 58(11):2755-2764.

14. Jiang J, Yu X. Fault-tolerant control systems: A comparative study between active and passive approaches. Annual Reviews in control 2012; 36(1):60-72.

15. Nazari R, Seron MM, Yetendje A. Invariant-set-based fault tolerant control using virtual sensors. IET control theory \& applications $2011 ; \mathbf{5}(9): 1092-1103$.

16. Zhang L, Boukas EK. $\mathrm{H} \infty$ control for discrete-time markovian jump linear systems with partly unknown transition probabilities. International Journal of Robust and Nonlinear Control 2009; 19(8):868-883.

17. Zhang H, Wang J, Shi Y. Robust h $\infty$ sliding-mode control for markovian jump systems subject to intermittent observations and partially known transition probabilities. Systems \& Control Letters 2013; 62(12):1114-1124.

18. de Souza CE. Robust stability and stabilization of uncertain discrete-time markovian jump linear systems. Automatic Control, IEEE Transactions on 2006; 51(5):836-841.

19. Wan Z, Kothare MV. An efficient off-line formulation of robust model predictive control using linear matrix inequalities. Automatica 2003; 39(5):837-846.

20. Zhang L, Boukas EK. Stability and stabilization of markovian jump linear systems with partly unknown transition probabilities. Automatica 2009; 45(2):463-468.

21. Ma S, Boukas EK. Guaranteed cost control of uncertain discrete-time singular markov jump systems with indefinite quadratic cost. International journal of robust and nonlinear control 2011; 21(9):1031-1045. 